\title{
Dois diluentes comerciais na criopreservação de espermatozoides do epidídimo de touros
}

Two commercial extenders for cryopreservation of epididymal bull sperm

\author{
Melina Andrea Formighieri Bertol ${ }^{*}$ Romildo Romualdo Weiss $^{\mathrm{I}}$ Aline Silva Fujita ${ }^{\mathrm{II}}$ \\ Luiz Ernandes Kozicki" Ana Claudia Machinski Rangel de Abreu ${ }^{\text {III }}$ João Filipi Scheffer Pereira ${ }^{\text {III }}$
}

RESUMO

A recuperação e a criopreservação de espermatozoides do epidídimo constituem alternativas viáveis para a preservação de material genético de animais valiosos. $O$ objetivo deste estudo foi comparar o desempenho de dois diluentes comerciais Botu-Bov ${ }^{\circledR}(B B)$ e Bovimix ${ }^{\circledR}(B V)$, sobre a viabilidade pós-descongelação de espermatozoides do epidídimo de touros Tabapuã (Bos taurus indicus) pós-castração. Os espermatozoides foram colhidos da cauda de 20 epidídimos utilizando a técnica de fluxo retrógrado, centrifugados e diluídos com $B B$ ou $B V$ para posterior criopreservação a $-196^{\circ} \mathrm{C}$. Após a descongelação, as amostras foram avaliadas utilizando a análise computadorizada (CASA) e por análises microscópicas para a determinação da integridade de membranas plasmáticas, acrossomal e morfologia espermática. A avaliação estatística dos dados foi realizada pela análise de variância (ANOVA) com o pós-teste de comparações múltiplas de Tukey-Kramer, com nível de significância $(P<0,05)$. Os resultados do movimento espermático avaliado pelo CASA, não diferiram para o diluente BB e BV. Também não foi observada diferença significativa entre os grupos no percentual de espermatozoides morfologicamente deformados, defeitos de acrossoma e espermatozoides com membrana plasmática íntegra após o descongelamento. Conclui-se que ambos os diluentes (BB e BV) são eficientes e podem ser utilizados na tecnologia do congelamento de espermatozoides colhidos da cauda do epidídimo de touros, não apresentando diferença na viabilidade espermática para os parâmetros estudados.

Palavras-chave: diluente, viabilidade espermática, epidídimo, análise computadorizada do sêmen (CASA).

\section{ABSTRACT}

Recovery and cryopreservation of epididymal sperm is a viable alternative for preservation of genetically valuable animals. The aim of this study was to verify and to compare the effect of two commercial extenders for conventional semen on postthawing viability of bovine epididymal sperm. For this purpose, the spermatozoa was recovered from the tail of 20 epididymis of Tabapuã bulls (Bos Taurus indicus) using retrograde flow method. After sperm recovery, the cells were centrifuged and divided for dilution with the diluents Botu-Bov ${ }^{\circledR}(B B)$ or Bovimix ${ }^{\circledR}$ (BV) for cryopreservation at $-196^{\circ} \mathrm{C}$. After thawing, all samples were evaluated using computer assisted sperm analysis (CASA), and by microscopic analysis for determination of integrity of plasma and acrossomal membrane and morphology. Statistical evaluation was performed by analysis of variance (ANOVA) with post-test for multiple comparisons, the Tukey-Kramer test, with significance level $(P<0.05)$. The results of the sperm movement for diluent $B B$ and $B V$ evaluated with CASA, showed no difference for both $(P>0.05)$. There was also no difference between the percentage of deformed sperm, acrosome defects and the sperm with intact plasma membrane after thawing with $B B$ or $B V$. We conclude that both extenders ( $B B$ and $B V$ ) are efficient and can be used for freezing sperm collected from the epididymis of bulls, showing no difference for all the parameters studied.

Key words: extender, sperm viability, epididymis, computer assisted sperm analysis (CASA).

\section{INTRODUÇÃO}

A recuperação e criopreservação de espermatozoides do epidídimo é uma ferramenta biotecnológica para recuperar material genético mesmo após a morte de animais de alto valor zootécnico ou ameaçados de extinção (KAABI et al., 2003). A criopreservação dos espermatozoides da cauda do epidídimo após a colheita possibilita seu

\footnotetext{
'Programa de Pós-graduação em Engenharia de Bioprocessos e Biotecnologia, Área de Concentração em Saúde Animal e Humana, Universidade Federal do Paraná (UFPR), 80035-050, Curitiba, PR, Brasil. E-mail: melbertol@hotmail.com. *Autor para correspondência.

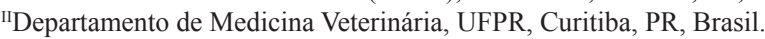

IIICentro de Ciências Agrárias e Ambientais, Pontifícia Universidade Católica do Paraná (PUC-PR), Curitiba, PR, Brasil.
} Recebido 27.05.13 Aprovado 10.02.14 Devolvido pelo autor 20.06.14 CR-2013-0747.R2 
aproveitamento de forma mais eficiente e econômica, pois permite a utilização por tempo indeterminado em programas de inseminação ou fecundação in vitro e não apenas logo após a morte do animal, como ocorre caso esse material não seja preservado (MARTINS et al., 2007). Alguns autores já relataram, com sucesso, a criopreservação de espermatozoides do epidídimo de bovinos (MARTINS et al., 2007; COSTA et al., 2011).

A utilização do sêmen bovino criopreservado apresenta vantagens em relação às demais formas de processamento, possibilitando a expansão da genética de diferentes touros para o comércio mundial, além de atuar na prevenção da disseminação de doenças passíveis de transmissão por meio de monta natural (VERBERCKMOES et al., 2005), entretanto, geralmente fornece menores taxas de fertilidade em relação ao sêmen fresco. Os principais fatores envolvidos nessa queda de fertilidade são as mudanças de temperatura durante o processamento, o estresse osmótico e tóxico representado pela exposição aos crioprotetores e a formação e dissolução de cristais de gelo extracelulares (WATSON, 2000).

O sucesso da congelação espermática depende da efetividade do processamento que atua no bloqueio completo dos processos metabólicos desenvolvidos pelas células espermáticas (VISHWANATH \& SHANNON, 2000), e a interação entre as células espermáticas e o meio diluidor representa um fator crucial para a preservação da integridade celular e sucesso da fertilização após o congelamento (CHAVEIRO et al., 2006). Os componentes essenciais dos meios de criopreservação são as substâncias iônicas e não iônicas responsáveis pela manutenção da osmolaridade e tamponamento, as fontes de lipoproteínas necessárias para a prevenção do choque frio, os crioprotetores como glicerol, etilenoglicol e DMSO, as fontes energéticas representadas por açúcares, como glicose e frutose, e os demais aditivos, tais como antibióticos, enzimas e detergentes (VISHWANATH \& SHANNON, 2000).

A coleta de espermatozoides diretamente da cauda do epidídimo representa uma alternativa promissora quando por algum motivo não é possível obter gametas através da ejaculação (JONES, 2004) ou quando sua preservação seja de suma importância. Não há no mercado nacional e internacional atualmente um diluente específico para a criopreservação de espermatozoides colhidos diretamente do epidídimo, nem protocolos exclusivos para o congelamento, o que garantiria maior sucesso dessa biotecnologia, assegurando melhores resultados no futuro. Nesse contexto, objetivou-se verificar o desempenho de dois diluentes comerciais existentes no mercado brasileiro para a criopreservação de sêmen convencional BotuBov $^{\circledR}$ e Bovimix ${ }^{\circledR}$ sobre a viabilidade (movimento, morfologia e integridade de membranas plasmáticas e acrossomal) pós-descongelação de espermatozoides colhidos do epidídimo de touros.

\section{MATERIAL E MÉTODOS}

No presente estudo, foram utilizados 20 epidídimos obtidos por orquiectomia bilateral de 10 touros da raça Tabapuã (Bos taurus indicus), com peso médio de $523 \mathrm{~kg}$ e circunferência escrotal média de $36,2 \pm 3,2 \mathrm{~cm}$. Os animais foram mantidos em sistema extensivo de produção e o manejo alimentar foi através de pastejo em brachiaria sp., suplementação mineral e água a vontade. A orquiectomia bilateral foi realizada em tronco de contenção com o animal em estação através da aplicação de anestésico local (cloridato de lidocaína $2 \%$ com vasoconstrictor), $10 \mathrm{~mL}$ em cada cordão espermático e $5 \mathrm{~mL}$ na região da incisão. Uma incisão com aproximadamente $5 \mathrm{~cm}$ de comprimento foi feita na região lateral de cada testículo, seccionando-se a pele e túnica dartos e a túnica vaginal até exposição do testículo e da túnica albúlgínea. Em seguida, o testículo foi tracionado e liberado do ligamento testicular, permitindo a manipulação do cordão espermático, no qual foi realizada a hemostasia e incisão final. O procedimento cirúrgico demorou em média 3,4 $\pm 1,9$ minutos por testículo.

Após a orquiectomia, o material retirado foi levado até o laboratório para manipulação. Num primeiro momento, procedeu-se à lavagem do complexo testículo-epidídimo com solução de Ringer-lactato aquecida a $37^{\circ} \mathrm{C}$ para remoção de sujidades e vestígios de sangue. Utilizando-se pinças hemostáticas e bisturi, o epidídimo foi separado do testículo e, em seguida, a região da cauda e o ducto deferente foram isolados do corpo e da cabeça do epidídimo para obtenção dos espermatozoides pela técnica de fluxo retrógrado, com adaptações para a espécie bovina (BERTOL, 2012).

Após a colheita, as amostras obtidas de cada cauda epididimária, vinte ao todo, foram divididas em duas frações e centrifugadas a $600 \mathrm{~g}$ por 10 minutos. Após a centrifugação, desprezou-se o sobrenadante, sendo o precipitado ressuspendido com os diluentes Botu-Bov ${ }^{\circledR}$ - BB (Botupharma, Botucatu, São Paulo, Brasil) ou Bovimix ${ }^{\circledR}$ - BV (Nutricell, Campinas, São Paulo, Brasil). O envase foi feito em palhetas de $0,25 \mathrm{~mL}$, lacradas com álcool polivinílico, numa concentração de $20 \times 10^{6}$ de espermatozoides com motilidade total por palheta e cada palheta 
foi identificada com o tipo de diluente utilizado. Após esta etapa, as palhetas foram submetidas à refrigeração em container de transporte de sêmen Botu-tainer $^{\circledR}$ (Biotech, Botucatu, SP, Brasil) para manutenção da temperatura em $5^{\circ} \mathrm{C}$ por período de equilíbrio de 3 horas.

Em seguida, procedeu-se à criopreservação em caixa isotérmica em vapor de nitrogênio líquido por um período de 20 minutos, através da alocação das palhetas em uma grade de metal posicionada $5 \mathrm{~cm}$ acima da coluna de nitrogênio líquido. Após esse período, as palhetas foram imersas diretamente em nitrogênio líquido $\left(-196^{\circ} \mathrm{C}\right)$ e, com auxílio de uma pinça hemostática, foram alojadas no compartimento superior e inferior de raques de metal, identificadas e transferidas para o botijão criogênico.

Para a avaliação, 20 palhetas de cada grupo (diluente BB e diluente BV), sendo uma palheta de cada partida, totalizando 20 amostras por grupo, foram descongeladas em banho-maria a $40^{\circ} \mathrm{C}$, por 20 segundos, e submetidas à análise computadorizada do movimento espermático - CASA (Hamilton Thorn Motility Analyser - HTMA - IVOS 12.3 - Hamilton Research - Beverly, MA, USA). Foram selecionados ao acaso três campos, observando-se os seguintes parâmetros: motilidade total (MT, \%); motilidade progressiva (MP, \%); velocidade de trajeto (VAP, $\mu \mathrm{ms}^{-1}$ ); velocidade progressiva (VSL, $\mu \mathrm{m} \mathrm{s}^{-1}$ ); velocidade curvilinear (VCL, $\mu \mathrm{m} \mathrm{s}^{-1}$ ); amplitude de deslocamento lateral de cabeça $(\mathrm{ALH}, \mu \mathrm{m})$; frequência de batimento espermático (BCF, Hz); retilinearidade (STR, \%); linearidade (LIN, \%); e rapidez (RAP, \%). Os ajustes do setup utilizados para realização da leitura do aparelho foram: configuração de análise (RAM), captura de imagem (frames por segundo: $60 \mathrm{~Hz}$, número de frames: 30), cela de detecção (contraste mínimo: 60; tamanho celular mínimo: 5 pix), padrões se houver $<5$ células móveis (tamanho celular: 5pix, intensidade celular: 55). Células em progressão (velocidade de trajeto: VAP: $75 \mu \mathrm{s}^{-1}$; lineariedade: STR: $80 \%$ ), células lentas: células lentas estáticas (VAP limite de corte de $21,9 \mu \mathrm{s}^{-1}$; VSL limite de corte de $6,0 \mu \mathrm{s}^{-1}$ ). Canais estáticos: intensidade (mínimo 0,25; máximo 1,50), tamanho (mínimo 0,60; máximo 8,00), alongamento (mínimo 0; máximo 95). Calibração (ampliação: 1,95), fonte de vídeo (frequência: $60 \mathrm{~Hz}$; campo: escuro), iluminação (intensidade: 2.400), fotômetro (baixo: 73, alto: 125). Câmara de análise (tipo: Makler; profundidade: $10.0 \mu \mathrm{m}$; posição: 14,3 milímetros; seleção dos campos: automático; temperatura de análise: $37^{\circ} \mathrm{C}$ ).

Foi retirada uma alíquota das amostras para a avaliação da morfologia espermática em microscopia de contraste de fase em aumento de 1000x sob imersão através de preparações úmidas, classificando-se 200 células por preparação, de acordo com BLOM (1973). Na análise dos dados, enfocaram-se espermatozoides com defeitos em acrossoma. Outro parâmetro avaliado neste estudo foi integridade total da membrana plasmática (IMP) dos espermatozoides através do uso de sondas fluorescentes, diacetato de carboxifluoresceina (DIC) e iodeto de propídio (IP), de acordo com o método descrito por HARRISON \& VICKERS (1990). As suspensões coradas foram depositadas entre lâmina e lamínula e observadas em aumento de 400x em microscópio de epifluorescência, em que 200 células lâmina foram avaliadas e distribuídas percentualmente (\%) nas seguintes classificações: Íntegros - acúmulo de DIC (fluorescência verde) ao longo da cabeça e flagelo, sem acúmulo de IP (fluorescência vermelha); Lesados - acúmulo de IP no núcleo celular, sem acúmulo de DIC ou acúmulo de fluorescência amarelada na região acrossomal. O total de espermatozoides com membranas íntegras (IMP) nas amostras foram classificados em dados percentuais.

Os dados obtidos no experimento inicialmente foram submetidos ao Teste de normalidade de Shapiro-Wilk e ao teste de KruskalWallis, com o pós-teste de Dunn. A avaliação estatística dos dados foi realizada pela análise de variância (ANOVA) com o pós-teste de comparações múltiplas de Tukey-Kramer. Para as análises, utilizouse nível de significância $(\mathrm{P}<0,05)$. Para execução da estatística, usou-se o software StatView (SAS Institute Inc., Cary, NC, EUA).

\section{RESULTADOS E DISCUSSÃO}

As injúrias causadas pelos componentes do meio diluidor (crioprotetores) estão entre os principais estresses que a célula espermática sofre durante o processo de congelamento (VISHWANATH \& SHANNON, 2000). Já está demonstrado que os diluentes de criopreservação de sêmen bovino exercem influência significativa sobre os parâmetros de congelabilidade espermática avaliados laboratorialmente (CRESPILHO, 2007), por isso escolher o diluente ideal é essencial para minimizar os danos causados e manter a capacidade de fertilização dos espermatozoides após o processo de criopreservação.

Quando comparamos dois diluentes existentes no mercado nacional para sêmen de touro na criopreservação de espermatozoides colhidos diretamente do epidídimo, encontramos resultados 
semelhantes (Tabela 1), não havendo diferença na média de motilidade total e motilidade progressiva, quando congelados com BB ou BV. Os valores de motilidade total encontrados estão inclusos nos valores aceitáveis para a utilização de sêmen congelado em inseminação artificial, e estiveram próximos aos relatados por outros autores na mesma espécie ao criopresevar espermatozoides do epidídimo (GOOVAERTS et al., 2006; PAPA et al., 2011). O movimento espermático representa uma das características mais importantes associadas ao potencial de fertilização de um espermatozoide, pois, com ausência de motilidade, a célula espermática é impossibilitada de realizar a migração no trato reprodutivo da fêmea até o encontro com o ovócito para a fecundação (LEMMA, 2011), sendo associado a casos de infertilidade. Os valores de motilidade progressiva encontrados foram baixos quando comparados ao recomendado pelo Colégio Brasileiro de Reprodução Animal após descongelação uma amostra de sêmen convencional (CBRA, 2008). Isso se deve ao fato de que, quando os espermatozoides são colhidos do epidídimo, possuem número expressivo de gotas citoplasmáticas posicionadas na porção distal da cauda, provocando movimentação circular ao invés de progressiva. Em contrapartida, o percentual de células em movimento é satisfatório em ambos os grupos, indicando a viabilidade dos

Tabela 1 - Avaliação comparativa entre diluentes comerciais Botu-bov $^{\circledR}$ e Bovimix ${ }^{\circledR}$ (média \pm desvio padrão) para os parâmetros espermáticos: MT (\%); MP (\%); VAP $\left(\mu \mathrm{ms}^{-1}\right)$; VSL $\left(\mu \mathrm{m} \mathrm{s}^{-1}\right)$; VCL $\left(\mu \mathrm{m} \mathrm{s}^{-1}\right)$; $\operatorname{ALH}(\mu \mathrm{m})$; BCF $(\mathrm{Hz})$; STR $(\%)$; LIN (\%) e RAP (\%) de espermatozoides do epidídimo de touros pósdescongelação.

\begin{tabular}{lcc}
\hline Parâmetro & Botu-bov $^{\circledR}(\mathrm{n}=20)$ & Bovimix $^{\circledR}(\mathrm{n}=20)$ \\
\hline MT $(\%)$ & $45,1 \pm 16,5^{\mathrm{a}}$ & $46,6 \pm 21^{\mathrm{a}}$ \\
MP $(\%)$ & $16,9 \pm 12,7^{\mathrm{a}}$ & $14,5 \pm 7,9^{\mathrm{a}}$ \\
VAP $\left(\mu \mathrm{m} \mathrm{s}^{-1}\right)$ & $64,52 \pm 10,2^{\mathrm{a}}$ & $62,15 \pm 11,4^{\mathrm{a}}$ \\
VSL $\left(\mu \mathrm{m} \mathrm{s}^{-1}\right)$ & $52,11 \pm 22,6^{\mathrm{a}}$ & $50,18 \pm 21,2^{\mathrm{a}}$ \\
VCL $\left(\mu \mathrm{m} \mathrm{s}^{-1}\right)$ & $120,15 \pm 41^{\mathrm{a}}$ & $114,12 \pm 39,7^{\mathrm{a}}$ \\
ALH $(\mu \mathrm{m})$ & $10,16 \pm 6,4^{\mathrm{a}}$ & $9,94 \pm 6,1^{\mathrm{a}}$ \\
BCF $(\mathrm{Hz})$ & $33,56 \pm 11,6^{\mathrm{a}}$ & $32,01 \pm 11^{\mathrm{a}}$ \\
STR $(\%)$ & $62,3 \pm 10,4^{\mathrm{a}}$ & $63,2 \pm 10,1^{\mathrm{a}}$ \\
LIN $(\%)$ & $36 \pm 3,4^{\mathrm{a}}$ & $36,2 \pm 2,6^{\mathrm{a}}$ \\
RAP $(\%)$ & $22,8 \pm 11,8^{\mathrm{a}}$ & $22,1 \pm 12,7^{\mathrm{a}}$ \\
\hline
\end{tabular}

MT - motilidade total (\%); MP - motilidade progressiva (\%); VAP - velocidade de trajeto $\left(\mu \mathrm{ms}^{-1}\right)$; VSL - velocidade linear $\left(\mu \mathrm{ms}^{-1}\right)$; VCL - velocidade curvilinear $\left(\mu \mathrm{ms}^{-1}\right)$; ALH deslocamento lateral da cabeça $(\mu \mathrm{m})$; BCF - frequência de batimento espermático $(\mathrm{Hz})$; STR - retilinearidade $(\%)$; LIN linearidade (\%) e RAP - rápidos (\%).

Letras iguais na mesma linha não diferem entre si $(\mathrm{P}>0,05)$. espermatozoides em um dos principais parâmetros passíveis de avaliação in vitro para predição da fertilidade in vivo.

Ainda na tabela 1 , são apresentados os demais parâmetros de movimentação espermática obtidos pela análise computadorizada. Em todos os parâmetros avaliados, não houve diferença entre os grupos $\mathrm{BB}$ e BV $(\mathrm{P}>0,05)$. Os dois diluentes utilizados neste estudo possuem composição semelhante, ambos são à base de gema de ovo, explicando resultados tão próximos, porém alguns constituintes não são revelados em suas fórmulas, gerando a necessidade de serem testados e confrontados. Quando se compara este trabalho com o desenvolvido por outro autor, observa-se que os resultados foram similares, uma vez que também não houve diferença na análise computadorizada do sêmen entre o diluente convencional TRIS e o Botu-bov ${ }^{\circledR}$ no congelamento de espermatozoides colhidos de epidídimos de touros (PAPA et al., 2011). Com isso, pode-se afirmar que, ao utilizar um diluente para congelamento de sêmen convencional de touro no congelamento de espermatozoides do epidídimo, os resultados não variam entre algumas fórmulas existentes no mercado, e que a composição de macro e micro elementos é capaz de suprir as necessidades básicas de proteção e manutenção das células espermáticas.

Por outro lado, em espécie diferente, MARTINS et al. (2012) viabilizaram a utilização do diluente nacional Bovimix ${ }^{\circledR}$ e do TRIS para a criopreservação de espermatozoides do epidídimo de cão e obtiveram melhores resultados com a utilização do Bovimix $^{\circledR}$ em relação ao TRIS na maioria dos parâmetros avaliados no CASA.

A média \pm desvio padrão da porcentagem de espermatozoides com alterações morfológicas (Tabela 2) pós-descongelamento não diferiram $(\mathrm{P}>0,05)$. A maior ocorrência de alteração morfológica foi composta pela presença de gotas citoplasmáticas distais, um defeito menor e característico dos espermatozoides colhidos diretamente da cauda do epidídimo (MARTINS et al., 2007; COSTA et al., 2011). A porcentagem de acrossomas lesados (DA) foi baixa e similar para os dois diluentes (BB e BV), demostrando que a alternância do meio diluidor não foi impactante nesse parâmetro assim como nos demais. A integridade da membrana plasmática dos espermatozoides não apresentou diferença significativa entre os diluentes BB e BV (Tabela 2), e ambos os meios foram capazes de conservar mais de $50 \%$ de espermatozoides com membranas íntegras pós-descongelamento, um bom indicativo de viabilidade, pois as membranas dos espermatozoides 
Tabela 2 - Avaliação comparativa entre diluentes comerciais Botu-Bov ${ }^{\circledR}$ e Bovimix ${ }^{\circledR}$ (média \pm desvio padrão) para a morfologia espermática, defeitos de acrossoma e integridade de membrana plasmática de espermatozoides do epidídimo de touros.

\begin{tabular}{lcccc}
\hline Diluente $(\mathrm{n}=20)$ & $-\mathrm{c}$ & DA (\%) & IMP (\%) \\
& Defeitos maiores & Defeitos menores & & \\
Botu-bov $^{\circledR}$ & $11,1 \pm 0,2$ & $32,8 \pm 4,7^{\mathrm{a}}$ & $9,6 \pm 1^{\mathrm{a}}$ & $51,2 \pm 13,5^{\mathrm{a}}$ \\
Bovimix $^{\circledR}$ & $10,3 \pm 0,6$ & $30,5 \pm 5^{\mathrm{a}}$ & $9,2 \pm 1,2^{\mathrm{a}}$ & $57,3 \pm 13,4^{\mathrm{a}}$ \\
\hline
\end{tabular}

Espermatozoides morfologicamente deformados (EMD, \%); defeitos de acrossoma, (DA, \%) e integridade de membrana plasmática (IMP, \%). Letras iguais na mesma coluna não diferem entre si $(\mathrm{P}>0,05)$.

são estruturas especializadas e exercem funções fundamentais na fecundação (ARRUDA et al., 2010). Os valores encontrados do percentual de espermatozoides com integridade de membrana foram semelhantes aos descritos por COSTA et al. (2011). Os estudos em criopreservação e fertilidade de espermatozoides do epidídimo de touros são escassos, indicando a necessidade de novos trabalhos para gerar dados para comparação e referência na utilização desse material biológico.

$\mathrm{O}$ alto custo na obtenção de animais e o consumo de tempo para as avaliações num programa de inseminação artificial, aliados à complexidade e à dependência da estrutura laboratorial imposta pela PIV (produção in vitro de embriões), tornaram inviável a realização de testes de fertilidade in vivo das amostras no período proposto para realização do estudo. Por outro lado, aliando-se a análise computadorizada de movimento espermático, morfologia espermática e integridade de membrana plasmática, que são atualmente algumas das técnicas mais modernas disponíveis para avaliação espermática, pode-se estimar o potencial de fertilização dos espermatozoides após criopreservação com Botu-bov ${ }^{\circledR}$ e Bovimix ${ }^{\circledR}$, uma vez que células espermáticas que apresentem bons resultados nesses testes provavelmente irão apresentar um bom desempenho em fertilização de oócitos e, em consequência, tornar possível o resgate de informação genética. É importante ressaltar que bons resultados de fertilidade in vivo são dependentes de outros fatores, dentre eles, as características da fêmea, do ambiente, e dos técnicos envolvidos no processo, não avaliados neste trabalho.

\section{CONCLUSÃO}

Conclui-se que os diluentes nacionais comerciais Botu-Bov ${ }^{\circledR}$ e Bovimix ${ }^{\circledR}$ constituem opções viáveis para a criopreservação de espermatozoides do epidídimo de touros, não apresentando diferença significativa entre si na viabilidade espermática (movimento, morfologia e integridade de membrana plasmática e acrossomal) pós-descongelação.

\section{COMITÊ DE ÉTICA E BIOSSEGURANÇA}

O presente trabalho foi aprovado pela Comissão de Ética no Uso de Animais do Setor de Ciências Agrárias (CEUA$\mathrm{SCA})$ da Universidade Federal do Paraná, sob o protocolo $\mathrm{n}^{-}$ $017 / 2013$.

\section{AGRADECIMENTOS}

Ao Conselho Nacional de Desenvolvimento Científico e Tecnológico (CNPq), pela concessão da bolsa de Doutorado.

\section{REFERÊNCIAS}

ARRUDA, R.L. et al. Técnicas para avaliação laboratorial da integridade estrutural e funcional do sêmen congelado de touros. Revista Brasileira Reprodução Animal, v.34, p.168-184, 2010. Disponível em <www.cbra.org.br>. Acesso em: jan. 2013.

BERTOL, M.A.F. Viabilidade de espermatozoides bovinos colhidos de epidídimos mantidos em temperatura ambiente de 18-20 ${ }^{\circ}$ C. 2012. 76f. Dissertação (Mestrado em Saúde Animal e Humana) - Curso de Pós-graduação em Engenharia de Bioprocessos e Biotecnologia, Universidade Federal do Paraná, PR.

BLOM, E. The ultrastructure of some characteristic sperm defects and a proposal for a new classification of the bull spermiogram. Nordisk Veterinaer Medicin, v.25, p.383-391, 1973. Disponível em: <http://www.ncbi.nlm.nih.gov/pubmed/4768226>. Acesso em: nov. 2012.

CHAVEIRO, A. et al. Improvement of parameters of freezing protocol for bull sperm using two osmotic Supports. Theriogenology, v.65, p.1875-1890, 2006. Disponível em: $<$ http://www.ncbi.nlm.nih.gov/pubmed/16310842>. Acesso em: nov. 2012. doi: 10.1016/j.theriogenology.2005.10.017.

COLÉGIO BRASILEIRO DE REPRODUÇÃO ANIMAL (CBRA). Manual para exame andrológico e avaliação de sêmen animal. 2.ed. 1998. 49p.

COSTA, P.M. et al. Birth of normal calves after artificial insemination using cryopreserved spermatozoa obtained from refrigerated epididymides of death bovine. Ciência Rural, v.4, p.869-874, 2011. Disponível em: <http://www.scielo.br/scielo. php?pid $=$ S0103-84782011000500022\&script $=$ sci_arttext $>$. Acesso em: jan. 2013. doi: http://dx. doi.org/10.1590/S010384782011005000045 .

CRESPILHO, A.M. Efeito do meio diluidor e da dose inseminante sobre a congelabilidade e fertilidade do sêmen 
bovino utilizado em programas de inseminação artificial em tempo-fixo (IATF). 2007. 124f. Dissertação (Mestrado em Reprodução Animal) - Programa de pós-graduação em Medicina Veterinária, Universidade Estadual Paulista, SP.

HARRISON, R.A.P.; VICKERS, S.E. Use of fluorescent probes to assess membrane integrity in mammalian spermatozoa. Journal Reproduction Fertility, v.88, n.1, p.343-352, 1990. Disponível em: <http://www.ncbi.nlm.nih.gov/pubmed/1690300>. Acesso em: dez. 2012. doi: 10.1530/jrf.0.0880343.

GOOVAERTS, I.G. et al. Evaluation of epididymal semen quality using the Hamilton-Thorne analyser indicates variation between the two caudae epididymides of the same bull. Theriogenology, v.15, p.323-30, 2006. Disponível em: <http://www.ncbi.nlm. nihgov/pubmed/16387353>. Acesso em: jan. 2012. doi:10.1016/j. theriogenology.2005.11.018.

JONES, R. Sperm survival versus degradation in the mammalian epididymis: a hypothesis. Biology of Reproduction, v.7, p.1405-1411, 2004. Disponível em: <http:// www.ncbi.nlm.nih. gov/pubmed/15215193>. Acesso em: dez. 2012. doi: 10.1095/ biolreprod.104.031252.

KAABI,M.etal.Effectofepididymishandlingconditionsonthequality of ram spermatozoa recovered post-mortem. Theriogenology, v.60, n.7, p.1249-1259, 2003. Disponível em: $<$ http://www.sciencedirect. com/science?_ob=MImg\&_imagekey=B6TCM-48NXDNY4D\&_ $\mathrm{cdi}=5174 \&$ user $=7430124 \&$ pii $=$ S 0093691 X03001390\& 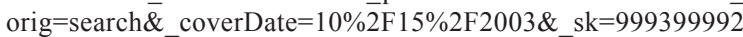 $\&$ view $=c \& w c h p=d G L b V l z z S k W A \& m d 5=5 \mathrm{~d} 21 \mathrm{f} 20482 \mathrm{f} 5530 \mathrm{f} 48$ 387b5860d77cf1\&ie=/sdarticle.pdf $>$. Acesso em: nov. 2012. doi: 10.1016/S0093-691X(03)00139-0.

LEMMA, A. Effect of cryopreservation on sperm quality and fertility. In: MANAFI, M. Artificial insemination in farm animals. Croatia: InTech, 2011. Cap.12, p.191-216.
MARTINS C.F. et al. Cryopreservation of epididymal bovine spermatozoa from dead animals and its uses in vitro embryo production. Animal Reproduction Science, v.101, p.326331, 2007. Disponivel em: <http://www.ncbi.nlm.nih.gov/ pubmed/17336472>. Acesso em: dez 2011. doi: 10.1016/j. anireprosci.2007.01.018.

MARTINS, M.I.M. et al. Comparison of two different extenders for cryopreservation of epididymal dog sperm. Reproduction Domestic Animal, v.47, p.293-294, 2012. Disponível em: <http:// www.ncbi.nlm.nih.gov/pubmed/23279522>. Acesso em: jan 2013. doi: 10.1111/rda.12042 10.1111/rda.12042.

PAPA, M.P. et al. Influence of two different extenders on cryopreservation of bovine epididymal sperm. Águas de São Pedro, SP. In: INTERNATIONAL CONFERENCE ON THE EPIDIDYMIS, 5., 2010. Anais... Animal Reproduction, v.8, n.34, p.104, 2011.

VISHWANATH, R.; SHANNON, P. Storage of bovine semen in liquid and frozen state. Animal Reproduction Science, v.62, p.23-53, 2000. Disponível em: <http:// elmu.umm.ac.id/file.php/1/ jurnal/A/Animal\%20Reproduction\%20Science/Vol62.Issue13. Aug2000/2026.pdf>. Acesso em: out. 2012.

WATSON, P.F. The causes of reduced fertility with cryopreserved semen. Animal Reproduction Science, v. 60-61, p.481492, 2000. Disponível em: <http://www.ncbi.nlm.nih.gov/ pubmed/10844218>. Acesso em: out. 2012.

VERBERCKMOES, S. et al. Comparison of three diluents for the storage of fresh bovine semen. Theriogenology, v.63, p.912-922, 2005. Disponível em: <http://www.ncbi.nlm.nih. gov/pubmed/15629807>. Acesso em: jan. 2012. doi: 10.1016/j. theriogenology.2004.05.011. 